\section{Case Reports in Ophthalmology}

Case Rep Ophthalmol 2020;11:348-355

DOI: 10.1159/000508326

Published online: July 14, 2020
(C) 2020 The Author(s)

Published by S. Karger AG, Basel www.karger.com/cop

\title{
How a Devastating Case of Acanthamoeba Sclerokeratitis Ended up with Serious Systemic Sequelae
}

\author{
Sophie Bataillie Rita Van Ginderdeuren Joachim Van Calster \\ Beatrijs Foets Heleen Delbeke \\ Ophthalmology, University Hospital Leuven, Leuven, Belgium
}

\section{Keywords}

Acanthamoeba keratitis · Acanthamoeba sclerokeratitis · Nodular scleritis · Enucleation

\begin{abstract}
A 35-year old soft contact lens wearer with a proven bilateral Acanthamoeba keratitis developed a nodular scleritis. Based on the stepladder approach described by lovieno et al. [Ophthalmology. 2014 Dec;121(12):2340-7], nonsteroidal anti-inflammatory drugs, methylprednisolone, and later azathioprine were added to the antiamoebic treatment. Unfortunately, there was further deterioration and an endophthalmitis developed. Unbearable pain and concerns of spread to the brain urged an enucleation. Histopathological examination confirmed Acanthamoeba cysts in the cornea, sclera, retina, choroid, and vitreous body. As a side effect of the immunosuppressive treatment, the patient developed myopathy, pulmonary aspergillosis, and an avascular necrosis of the hip. Scleritis is a devastating complication of Acanthamoeba keratitis with a poor prognosis and a high enucleation rate. Acanthamoeba sclerokeratitis is, due to cyst-free biopsies, mostly assigned to an immune-mediated mechanism, justifying the use of immunosuppressive treatment. Scleritis in our case contributed to the extracorneal spread of Acanthamoeba. Our case is the first documented extracorneal spread of Acanthamoeba without previous surgery. Extracorneal spread of Acanthamoeba should be considered, even in the case of false-negative biopsies. We strongly recommend serial sections of the retrieved scleral specimen in case of negative histopathological examination to exclude an infection. Even when an immune-mediated scleritis is suspected, systemic immunosup-
\end{abstract}




\section{Case Reports in Ophthalmology}

Case Rep Ophthalmol 2020;11:348-355

DOI: $10.1159 / 000508326$

C 2020 The Author(s). Published by S. Karger AG, Basel www.karger.com/cop

Bataillie et al.: How a Devastating Case of Acanthamoeba Sclerokeratitis Ended up with Serious Systemic Sequelae

pressive treatment should always be used with the greatest caution. Awareness of the side effects and monitoring by an experienced physician is mandatory.

\section{Introduction}

Acanthamoeba is a protozoan, having a life cycle consisting of trophozoite and cyst stages. The trophozoite is the infectious form causing corneal ulcers, especially in contact lens wearers. These trophozoites change into a double-walled dormant cystic form whenever the environment is hostile. It is in particular this double wall that makes the treatment of this infection so difficult and prolonged. Research proved that a delay of $>3$ weeks between diagnosis and symptoms worsen the outcome [1,2]. Acanthamoeba scleritis is a rare and painful complication of Acanthamoeba keratitis, affecting 10-18\% of patients [3-5]. The pathogenesis of Acanthamoeba sclerokeratitis is still poorly understood, and it is unclear whether it is immune-mediated or real spread of the infection causes this painful evolution [6]. This uncertainty makes it very difficult to treat, resulting in poor outcome.

We report a patient with histopathologically proven Acanthamoeba sclerokeratitis and uncontrollable endophthalmitis. The immunosuppressive agents used to control his sclerokeratitis led to severe secondary complications.

\section{Case Report}

A healthy 35-year-old soft contact lens wearer presented with bilateral peripheral stromal infiltrates over $360^{\circ}$ causing a foreign body sensation. At presentation, his vision was 0.3 ( $\log$ MAR) in both eyes and he had already been on topical corticosteroids for 1 week. Corneal scrape with histopathological examination and an agar enriched with Escherichia coli were both positive for Acanthamoeba. Topical treatment with polyhexamethylene biguanide $0.02 \%$ and propamidine isethionate $0.1 \%$ once every hour, continued overnight for the first $24 \mathrm{~h}$, moxifloxacine $0.5 \%$ six times a day, and atropine $1 \%$ twice a day was started. Under this treatment, the right eye improved rather quickly to a vision of 0.8 (logMAR), but the left eye deteriorated. After 15 days of intense antiamoebic treatment, the patient presented with keratic precipitates and anterior chamber inflammation. Topical prednisolone $1 \%$ four times a day was added to the treatment. The topical corticosteroids improved the inflammation, but recurrence was noticed after tapering down. One month after the initial diagnosis, propamidine isethionate was replaced by chlorhexidine $0.02 \%$ due to lack of improvement. Furthermore, based on the knowledge that $10-23 \%$ of Acanthamoeba keratitis cases may be polymicrobial or coinfected with herpes simplex virus, oral acyclovir was added [1]. After 3 months of intensive treatment, unbearable pain and marked conjunctival injection made us stop all drops for 2 days to exclude treatment-induced toxicity and to repeat the corneal scrape for histopathological examination, culture, and PCR. After stop of the treatment there was no improvement and the culture came back positive for yeast (unspecified). Amphotericin B drops $0.15 \%$ six times a day and oral itraconazole $100 \mathrm{mg}$ was added to the antiamoebic treatment of chlorhexidine $0.02 \%$, Desomedine $0.1 \%$, and polyhexamethylene biguanide $0.02 \%$, all six times a day, and the oral antiviral treatment.

Five months after the initial presentation, the patient was in severe pain, and an anterior scleritis with a painful nodule was diagnosed on ultrasound (Fig. 1). An immune-mediated scleritis due to Acanthamoeba keratitis was suspected (Fig. 2). Based on the stepladder 


\section{Case Reports in Ophthalmology}

Case Rep Ophthalmol 2020;11:348-355

DOI: $10.1159 / 000508326$

(C) 2020 The Author(s). Published by S. Karger AG, Basel www.karger.com/cop

Bataillie et al.: How a Devastating Case of Acanthamoeba Sclerokeratitis Ended up with Serious Systemic Sequelae

approach described by Iovieno et al. [5], oral ibuprofen $600 \mathrm{mg}$ three times a day, topical nepafenac $0.1 \%$ once a day and, in a later stage, intravenous solumedrol $1 \mathrm{~g}$ for 3 days followed by methylprednisolone orally could not improve his scleritis. Azathioprine $150 \mathrm{mg}$ a day was added to the treatment. A few days later, a new epithelial defect and corneal edema developed. Repeat histopathological examination reported Acanthamoeba cysts. A serious anterior and posterior chamber inflammation emerged under high-dose topical antiamoebic treatment in combination with the azathioprine and methylprednisolone. Because of the uncontrollable intraocular inflammation and a doubtful result on an earlier vitreous biopsy, a three-port hybrid 27- to 23-gauge vitrectomy combined with lensectomy and Siluron ${ }^{\circledR} 2000$ oil filling of the eye was performed. Retinal necrosis and edema of the optic disc became visible during surgery. An MRI of the brain did not show intracranial spread of the infection, but the unbearable pain in combination with the fear of spread to the brain made us perform an enucleation of the left eye.

Our immunocompetent patient, who was a passionate long-distance runner at diagnosis, developed a series of adverse events due to the immunosuppressive therapy that he took for 3 months. Besides anemia and elevated lactate dehydrogenase, thoracic imaging showed multiple lung nodules 4 months after start of the immunosuppressive treatment. Biopsy of these nodules showed hyphae, compatible with Aspergillus pneumonia, most probably related to the immunosuppressive treatment. In addition to this, he experienced severe myopathy with muscle weakness and atrophy of the proximal muscles in the lower limbs due to the oral steroids used. Two years after the enucleation, the patient presented with pain in his right hip and the diagnosis of avascular necrosis of the hip, a known complication of high-dose steroids, was made [7].

Histopathological examination of the enucleated eye showed a cornea with Acanthamoeba cysts in the stroma and decreased endothelial cells. The sclera was infiltrated by lymphocytes and histiocytes in combination with Acanthamoeba cysts in the superficial and deeper layers. Intraocularly, the vitreous body, the retina, the choroid, and even the subretinal and subchoroidal layers were infiltrated by lymphocytes, macrophages, and histiocytes. In the retina, an excessive amount of Acanthamoeba cysts were present. The optic nerve was atrophic, but without inflammation and Acanthamoeba cysts (Fig. 3).

\section{Discussion}

The increasing incidence of Acanthamoeba keratitis over the last 10 years makes it even more important to create awareness as regards potential serious sequelae [8]. The diagnosis of Acanthamoeba sclerokeratitis is often delayed because of the masking effect of the keratitis. Scleritis should be suspected in cases of severe pain, globe tenderness, engorged episcleral vessels, anterior uveitis, and scleral thickening on ultrasonography [3]. It is most commonly anterior and can be either diffuse or nodular. All published case reports with documented extracorneal spread of the Acanthamoeba species, including ours, had nodular scleritis. Presentation of a nodular scleritis should alert the clinician that an infectious origin is more likely, so prudence when starting immunosuppressive treatment is in order.

The pathogenesis of Acanthamoeba scleritis is still poorly understood. Both spread of the infection and an autoimmune reaction can be the underlying cause. For a very long time there was only one documented scleral invasion on histological report [6], then in 2009 another case report with Acanthamoeba cysts in the limbal region was published [9]. Arnalich-Montiel et al. [10] reported 2 cases of nodular Acanthamoeba sclerokeratitis after penetrating and 


\section{Case Reports in Ophthalmology}

Case Rep Ophthalmol 2020;11:348-355

DOI: $10.1159 / 000508326$

C 2020 The Author(s). Published by S. Karger AG, Basel www.karger.com/cop

Bataillie et al.: How a Devastating Case of Acanthamoeba Sclerokeratitis Ended up with Serious Systemic Sequelae

tectonic keratoplasty. Recently in 2017 and 2018, two more cases of Acanthamoeba spread throughout the globe were published $[11,12]$. All case reports with documented extracorneal spread of Acanthamoeba had had previous intraocular surgery. Our case proves that extracorneal dissemination of Acanthamoeba is possible in longstanding disease, even without previous intraocular surgery.

Most published case reports of Acanthamoeba-related scleritis showed a purely granulomatous inflammation without cysts on histopathological examination, presuming an immune-mediated mechanism [13-15]. One should consider the possibility that the Acanthamoeba cysts were not in the examined histopathological sections, highlighting the importance of serial sections when histopathological examination is not able to show any cysts.

Treatment of Acanthamoeba sclerokeratitis is in most cases successful with the association of topical nonsteroidal anti-inflammatory drugs and topical corticosteroids to the antiamoebic treatment. In some cases, as in ours, it can be very challenging and systemic immunosuppression, even in combination with penetrating keratoplasty, might be necessary to control tissue destruction and pain [4].

We used the stepladder approach described by Iovieno et al. [5]. Topical nonsteroidal anti-inflammatory drugs and steroids are advised as first-line treatment. In case of insufficient response, oral steroids should be added. Unless there is a full response within 1 week, they advise to add steroid-sparing agents such as cyclosporine, azathioprine, or mycophenolate to avoid long-term steroid side effects. In selected cases of severe scleral inflammation, intravenous methylprednisolone or a combination of cyclosporine with mycophenolate or azathioprine can be used. When introducing immunosuppressive treatment, it is recommended to start oral itraconazole or voriconazole. Good results with cyclophosphamide and anti-TNF therapy were described by Igras and Murphy [16], but the side effects of cyclophosphamide, such as severe nausea, vomiting, loss of appetite, hair loss, and even cancer, should be kept in mind.

Our case proves that even when a correct stepladder approach is used, prognosis can be devastating and an enucleation sometimes emerges as the only solution. Clinicians should be aware of the risk of myopathy, fungal infections, and avascular necrosis of the femoral head when high-dose corticosteroids and other immunosuppressive agents are prescribed. When avascular necrosis of the femoral head is suspected, prompt referral to an orthopedic surgeon is necessary, as early diagnosis and treatment yields a better prognosis [7]. In general, patients under immunosuppressive treatment need follow-up by an experienced physician with regular physical and hematological examinations [4].

\section{Conclusion}

Scleritis is a devastating complication of Acanthamoeba keratitis with a poor prognosis and a high enucleation rate of $6 \%$ [5]. The pathogenesis of scleritis is still poorly understood. Multiple case reports with negative histopathological examination assume an immune-mediated cause and suggest immunosuppressive treatment to improve symptoms and to reduce the enucleation rate. In contrast, our case shows that extracorneal spread of Acanthamoeba cysts is possible, even without previous intraocular surgery. In case of negative histopathological examination, serial sections should be looked at to exclude an infection. Systemic immunosuppressive treatment, even with prophylactic antiamoebic and antifungal agents, should always be used with the greatest caution. Awareness of the side effects is important 


\section{Case Reports in Ophthalmology}

and an experienced physician should monitor these patients with regular physical and hematological examination.

\section{Statement of Ethics}

The research was conducted ethically in accordance with the World Medical Association Declaration of Helsinki. The patient gave written informed consent for publication of his case (including publication of images). The study protocol was approved by the Committee on $\mathrm{Hu}-$ man Research at the KU Leuven. Information revealing the patient's identity was avoided.

\section{Conflict of Interest Statement}

The authors have no conflicts of interest to declare.

\section{Funding Sources}

None.

\section{Author Contributions}

S. Bataillie collected the information and wrote the manuscript. R. Van Ginderdeuren diagnosed the extracorneal spread of the Acanthamoeba cysts. Furthermore, she provided the pictures and description of the histopathological examination. H. Delbeke, B. Foets, and J. Van Calster were the physicians treating the patient and helped in writing and improving the article.

\section{References}

1 Dart JK, Saw VP, Kilvington S. Acanthamoeba keratitis: diagnosis and treatment update 2009. Am J Ophthalmol. 2009 Oct;148(4):487-499.e2.

2 Juárez MM, Tártara LI, Cid AG, Real JP, Bermúdez JM, Rajal VB, et al. Acanthamoeba in the eye, can the parasite hide even more? Latest developments on the disease. Cont Lens Anterior Eye. 2018 Jun;41(3):24551.

3 Mannis MJ, Tamaru R, Roth AM, Burns M, Thirkill C. Acanthamoeba sclerokeratitis. Determining diagnostic criteria. Arch Ophthalmol. 1986 Sep;104(9):1313-7.

4 Lee GA, Gray TB, Dart JK, Pavesio CE, Ficker LA, Larkin DF, et al. Acanthamoeba sclerokeratitis: treatment with systemic immunosuppression. Ophthalmology. 2002 Jun;109(6):1178-82.

5 Iovieno A, Gore DM, Carnt N, Dart JK. Acanthamoeba sclerokeratitis: epidemiology, clinical features, and treatment outcomes. Ophthalmology. 2014 Dec;121(12):2340-7.

6 Dougherty PJ, Binder PS, Mondino BJ, Glasgow BJ. Acanthamoeba sclerokeratitis. Am J Ophthalmol. 1994 Apr;117(4):475-9.

7 Cohen-Rosenblum A, Cui Q. Osteonecrosis of the Femoral Head. Orthop Clin North Am. 2019 Apr;50(2):13949.

8 Randag AC, Verkerk S, Van Goor AT, Van Rooij J, Wisse RPL, Saelens IEY, et al. Acanthamoebe keratitis in the Netherlands. Acta Ophthalmol. 2018;96(Suppl. A260):3.

9 Ebrahimi KB, Green WR, Grebe R, Jun AS. Acanthamoeba sclerokeratitis. Graefes Arch Clin Exp Ophthalmol. 2009 Feb;247(2):283-6. 


\section{Case Reports in Ophthalmology}

\begin{tabular}{l|l}
\hline DOI: $10.1159 / 000508326$ & ○ 2020 The Author(s). Published by S. Karger AG, Basel
\end{tabular}
www.karger.com/cop

Bataillie et al.: How a Devastating Case of Acanthamoeba Sclerokeratitis Ended up with Serious Systemic Sequelae

10 Arnalich-Montiel F, Jaumandreu L, Leal M, Valladares B, Lorenzo-Morales J. Scleral and intraocular amoebic dissemination in Acanthamoeba keratitis. Cornea. 2013 Dec;32(12):1625-7.

11 Mammo Z, Almeida DR, Cunningham MA, Chin EK, Mahajan VB. Acanthamoeba endophtalmitis after recurrent keratitis and nodular scleritis. Retin Cases Brief Rep. 2017;11(2):180-2.

12 Baker MS, Maltry AC, Syed NA, Allen RC. Orbital implant exposure after Acanthamoeba panophthalmitis. Am J Ophthalmol Case Rep. 2018 Jan;10:48-50.

13 Kato K, Hirano K, Nagasaka T, Matsunaga K, Takashima Y, Kondo M. Histopathological examination of Acanthamoeba sclerokeratitis. Clin Ophthalmol. 2014 Jan;8:251-3.

14 Chatterjee S, Agrawal D, Vemuganti GK. Granulomatous inflammation in Acanthamoeba sclerokeratitis. Indian J Ophthalmol. 2013 Jun;61(6):300-2.

15 Shi L, Hager T, Fries FN, Daas L, Holbach L, Hofmann-Rummelt C, et al. Reactive uveitis, retinal vasculitis and scleritis as ocular end-stage of Acanthamoeba keratitis: a histological study. Int J Ophthalmol. 2019 Dec; 12(12):1966-71.

16 Igras E, Murphy C. Use of multiple immunosuppressive agents in recalcitrant Acanthamoeba scleritis. BMJ Case Rep. 2015 Apr;2015:bcr2014208536.

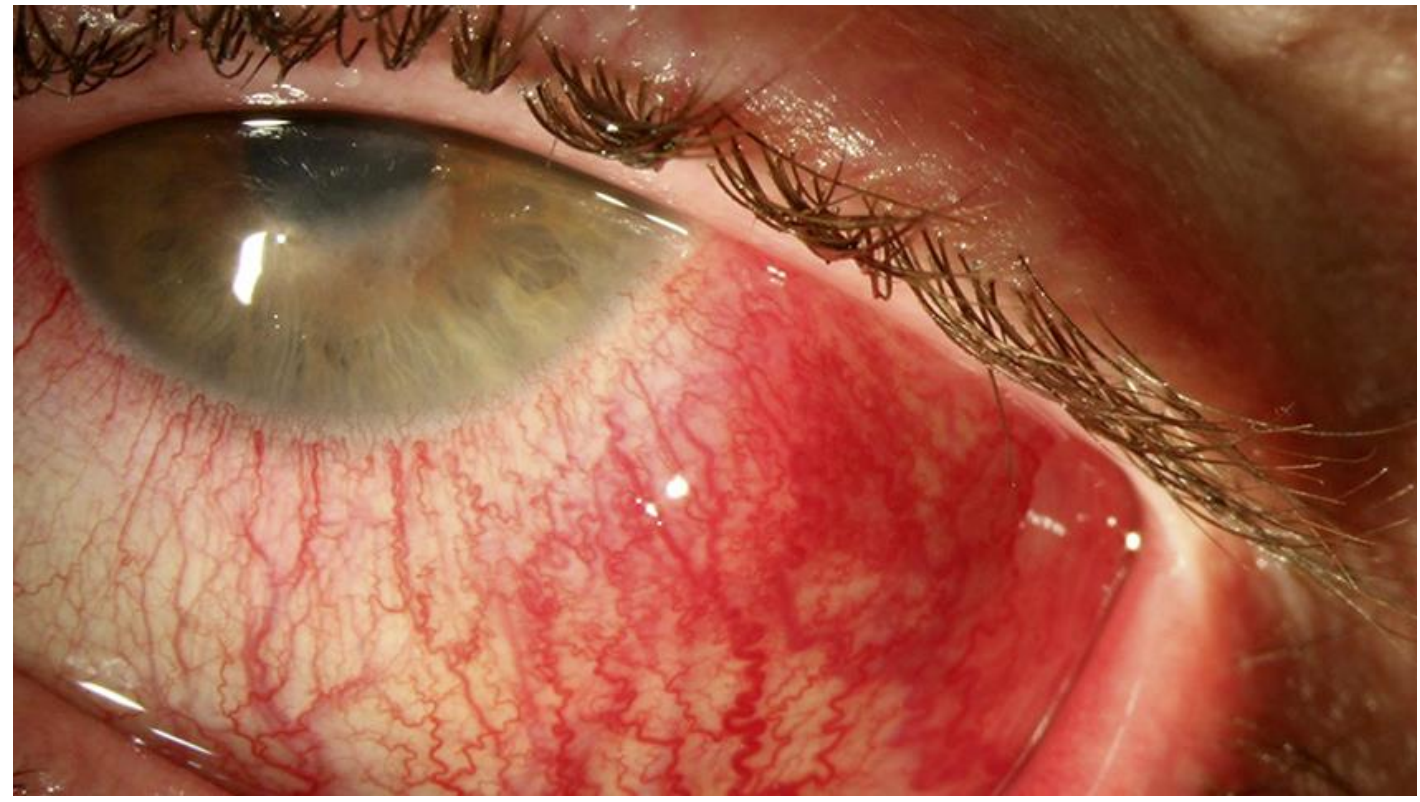

Fig. 1. Anterior scleritis of the left eye with a painful nodule. 
Case Reports in Ophthalmology
Case Rep Ophthalmol 2020;11:348-355

DOI: $10.1159 / 000508326$

(c) 2020 The Author(s). Published by S. Karger AG, Basel www.karger.com/cop

Bataillie et al.: How a Devastating Case of Acanthamoeba Sclerokeratitis Ended up with Serious Systemic Sequelae

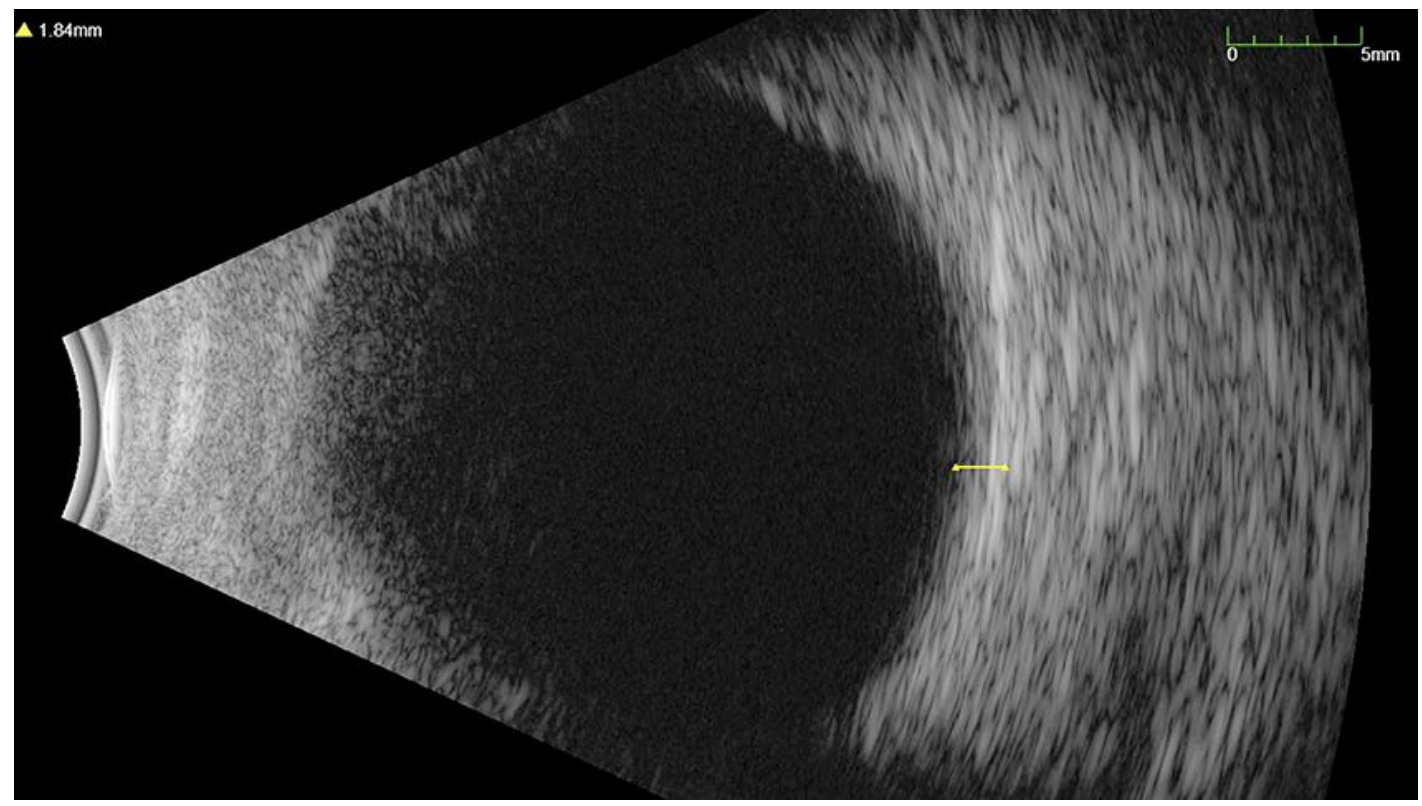

Fig. 2. Localized scleral (yellow arrow) and choroidal swelling in the inferotemporal sector of the left eye. 


\section{Case Reports in Ophthalmology}
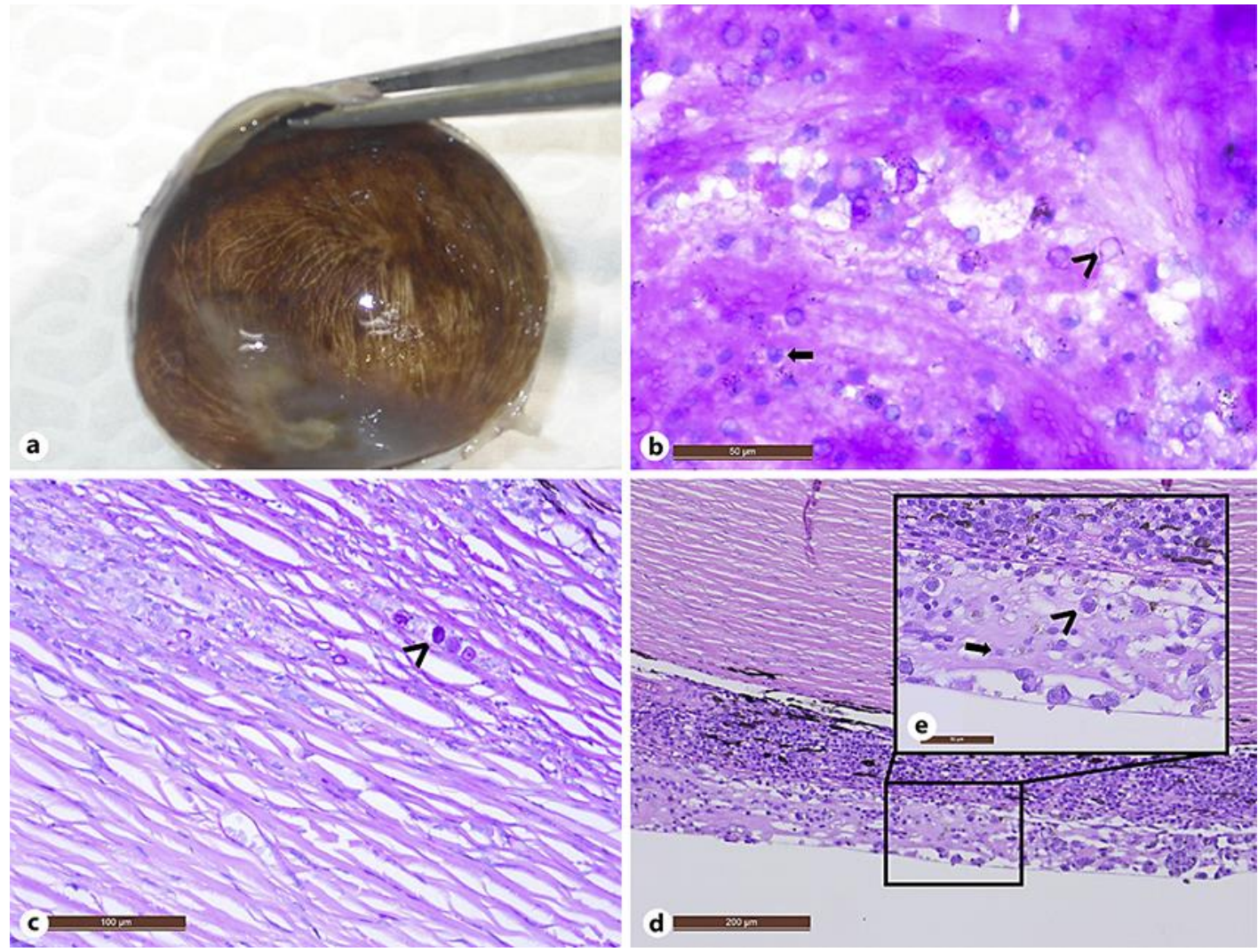

Bataillie et al.: How a Devastating Case of Acanthamoeba Sclerokeratitis Ended up with Serious Systemic Sequelae www.karger.com/cop

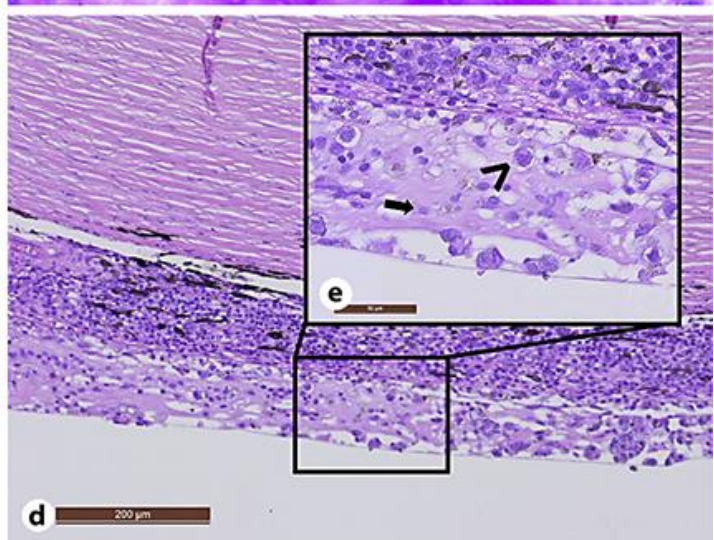

Fig. 3. Anatomopathological examination of the enucleated left eye (a) showed, after periodic acid-Schiff staining, multiple inflammatory cells $(\longrightarrow)$ and Acanthamoeba cysts ( $>$ ) in the vitreous (b), sclera (c), and retina (e). d The retina, choroidea, and sclera with magnification of the atrophic retina (e). 